\title{
APICO-BASAL DIAMETERS OF THE LUNGS AND MEDIASTINAL SHIFT IN PHRENIC NERVE CRUSH AND PNEUMOPERITONEUM THERAPY: A STUDY OF EIGHTY CASES
}

BY

\author{
WALLACE FOX \\ From Preston Hall Hospital, Aylesford, Kent
}

The objects of this paper are (a) to report on a study of mediastinal shift and apico-basal diameters of the lungs in a series of 80 patients undergoing phrenic nerve crush and pneumoperitoneum therapy for pulmonary tuberculosis: $(b)$ to discuss the mechanism and therapeutic significance of the mediastinal shift.

Although studies of mediastinal shift following phrenic nerve paralysis alone have been reported by Nehil and Alexander (1933) and Hansen and Maly (1934), no previous study of mediastinal shift following phrenic nerve crush and pneumoperitoneum therapy has been found in a search of the literature.

\section{Present Study}

The series consisted of 80 patients undergoing phrenic nerve crush and pneumoperitoneum therapy for pulmonary tuberculosis. All were adults, 42 being men and 38 women. There were 40 patients with left, and 40 with right, phrenic nerve crush. The average age of the patients was 27.3 years, the range being 18 to 47 years. Three patients were under 20,55 in the $20-29,18$ in the $30-39$, and four in the 40-49 age-groups.

In each case a radiograph before treatment was begun, and a comparable radiograph when the phrenic nerve crush and pneumoperitoneum therapy was fully established, were selected for the measurement of the reduction of the apico-basal diameter and the mediastinal shift. In selecting the radiographs, particular care was taken to avoid errors of rotation, or of forward and backward tilt of the patient. All the films were postero-anterior radiographs taken in full inspiration with the patient erect, the tube distance from the film being six feet. In investigations of the apico-basal diameters of the lungs, it has always to be assumed that the depth of inspiration is identical at the time of exposure of the two radiographs selected for the measurements. This introduces an unavoidable source of error, but by virtue of the greatly restricted movement of the paralysed hemidiaphragm this error is smaller on the paralysed side than on the unparalysed side.

\section{SElection OF Material}

The comparable radiographs were selected bearing in mind that the shorter the interval between them the greater was the likelihood that any mediastinal shift detected would be due to the therapy rather than to alterations in the disease. 
Patients with a scoliosis, or a pneumothorax or thoracoplasty on either side were excluded as these are factors which materially alter the position of the mediastinum. The therapy itself does not produce scoliosis. Radiographs with indefinite cardiac outlines were also excluded.

Even after a careful selection of comparable radiographs and the exclusion of unsuitable cases, measurements of mediastinal shift are difficult for several reasons. First, in addition to the lateral shift of the mediastinum, there is also an upward movement (Figs. 1 and 2) which is often of a very marked degree on the side of the phrenic nerve crush. The significance of this will be discussed later. Second, the mediastinum and the lateral chest wall are not parallel flat planes, so that the distance of the mediastinum from the chest wall varies in different parts of the chest. Thus, it is largest in the middle of the chest and smaller below, where the cardiac bulges occur, and also above, where the chest narrows towards the thoracic inlet. Third, the heart-shadow alters its shape considerably during the treatment and the heart is often considerably rotated, especially after a left phrenic nerve crush and pneumoperitoneum (Figs. 3 and 4). This cardiac rotation has been studied in the course of electrocardiographic investigations of the heart during this treatment by Agnello (1938), by Elwood, Piltz, and Potter (1939), and by Benatt and Berg (1945).

However, despite these variable factors, the radiographs have revealed a mediastinal shift, and a means of measurement has been selected which, although not exact, is an index of its degree.

\section{Method of Measurement}

Apico-basal Diameters.-The measurement of the apico-basal diameter was made by taking the vertical distance between the plane passing through the upper border of the transverse process of the first thoracic vertebra and the highest point on the diaphragmatic contour as seen on the comparable postero-anterior radiographs.

Mediastinal Shift.-The horizontal distance between the sides of the vertebral bodies and the point of maximum bulge on the cardiac outline on the unparalysed side was measured before the beginning and after the full establishment of the treatment. The difference between these two distances was taken to be the measurement of mediastinal shift. This method of measurement gave a fixed bony point and a moving soft tissue point comparable with the bony and mobile points in the measurement of the apico-basal diameter.

\section{RESULTS}

Left Phrenic Paralysis.-An analysis of the figures for the 40 patients with a left phrenic nerve crush and pneumoperitoneum showed that the average diaphragmatic rise produced on the left side was $8.1 \mathrm{~cm}$. (range $15.5-2.2 \mathrm{~cm}$.).

The average reduction of the apico-basal diameter on the left side was $33.0 \%$ (range 57.2\%-9.8\%).

The average diaphragmatic rise produced on the right side by the pneumoperitoneum alone was $2.6 \mathrm{~cm}$. (range $7.0-0 \mathrm{~cm}$.). The average reduction of the right apico-basal diameter was $11.1 \%$ (range $25.5 \%-0$ ).

The average mediastinal shift to the right was $1.5 \mathrm{~cm}$. (range $8.5-0 \mathrm{~cm}$.). 

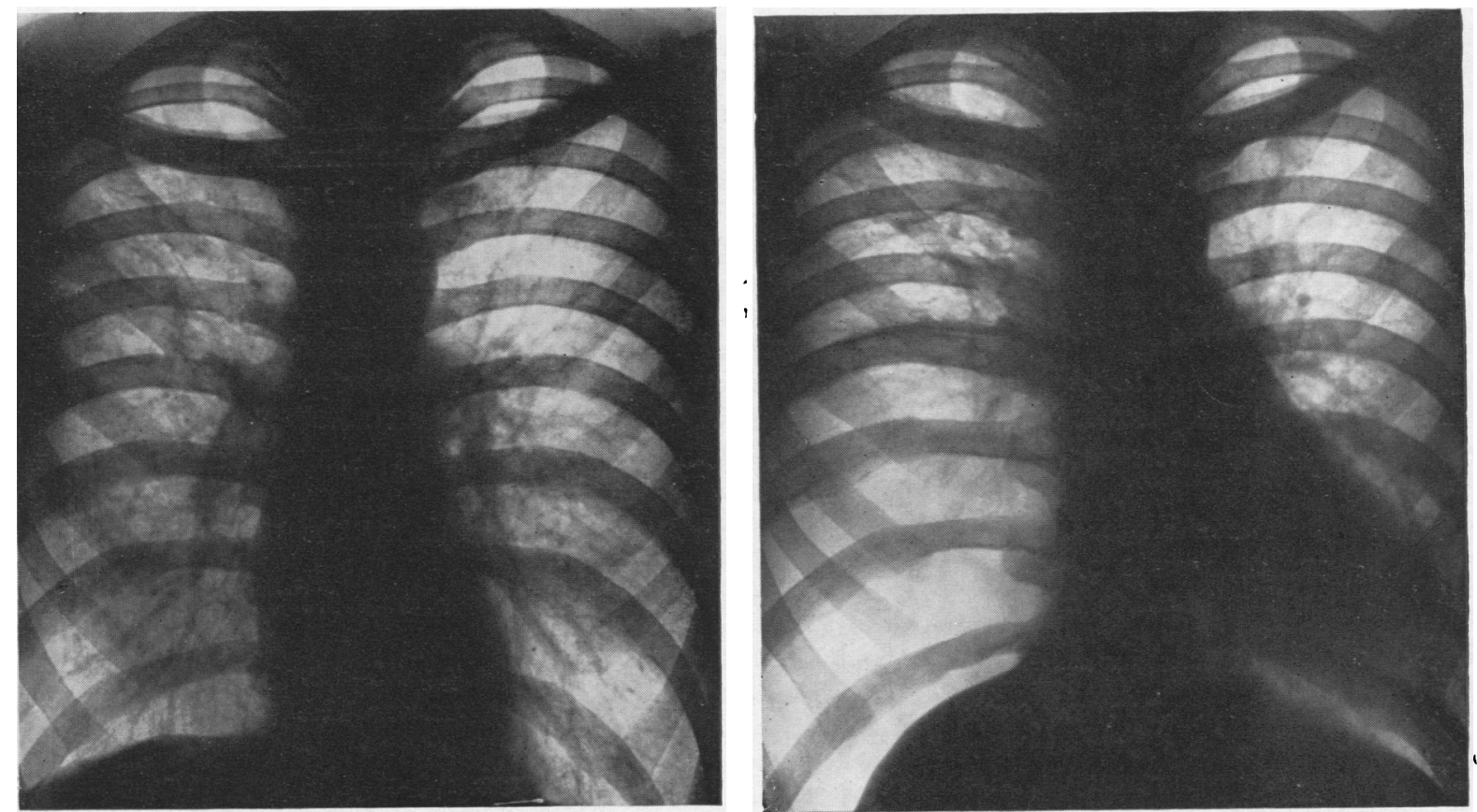

FIGs. 1 and 2.-Radiographs showing mediastinal shift to the left following a right phrenic nerve crush and pneumoperitoneum. The upward movement of the mediastinum on the paralysed side and the manner in which the intra-abdominal air lies lateral to the lower mediastinum is well demonstrated.
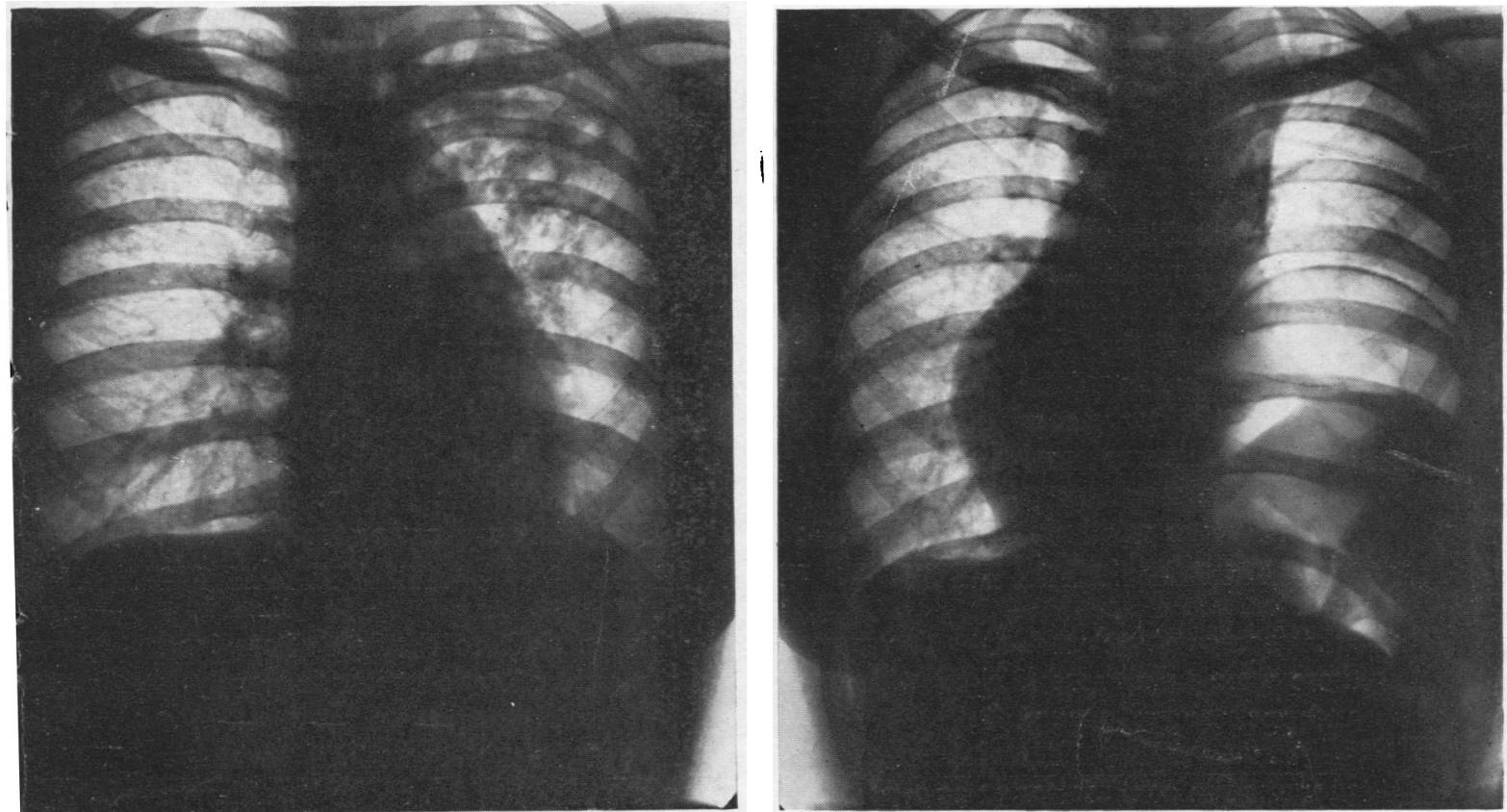

FIGs. 3 and 4.- Radiographs showing a striking degree of cardiac rotation and shift to the right following a left phrenic crush and pneumoperitoneum. The manner in which the intraabdominal air lies lateral to the lower mediastinum is demonstrated. 
In order to relate the mediastinal shift to the width of the hemithorax, the mediastinal shift was expressed as a percentage of the width of the hemithorax, at the level of the point of maximum bulge on the cardiac outline in the radiograph taken before treatment was begun. Using this method, the average reduction of the width of the right hemithorax after left phrenic crush and pneumoperitoneum was $11.0 \%$ (range $64.9 \%-0$ ).

Right Phrenic Paralysis.-An analysis of the figures for the 40 patients with a right phrenic nerve crush and pneumoperitoneum showed that the average diaphragmatic rise produced on the right side was $7.7 \mathrm{~cm}$. (range $13.6-4.0 \mathrm{~cm}$.).

The average reduction of the apico-basal diameter was $33.2 \%$ (range $51.9 \%-18.6 \%)$.

The average diaphragmatic rise produced on the left side by the pneumoperitoneum alone was $2.4 \mathrm{~cm}$. (range $6.0-0 \mathrm{~cm}$.).

The average reduction of the left apico-basal diameter was 9.4\% (range $23.8 \%-0)$.

The average mediastinal shift to the left was $1.6 \mathrm{~cm}$. (range $4.0-0 \mathrm{~cm}$.).

The average reduction of the width of the left hemithorax was $11.8 \%$ (range $28.2 \%-0$ ).

By comparing Nehil and Alexander's (1933) measurements of diaphragmatic elevation in a series of 214 patients treated by phrenic nerve paralysis alone with the figures in the present series, certain conclusions can be drawn concerning the relative effects of a pneumoperitoneum, a phrenic nerve paralysis, and the combined phrenic paralysis and pneumoperitoneum.

With pneumoperitoneum alone, the rise of the unparalysed hemidiaphragm is materially less than that caused by phrenic paralysis. In addition, the pumping action of the diaphragm, although diminished (Trimble, Eaton, Crenshaw, and Gourley, 1948) continues in the presence of the pneumoperitoneum. Hence, the effect of the pneumoperitoneum on the unparalysed side seems both statically and dynamically inferior to a phrenic paralysis as a collapse measure.

In a combined phrenic nerve crush and pneumoperitoneum, on the other hand, the diaphragmatic rise is considerably greater than that produced by phrenic paralysis alone, so that it is statically superior to phrenic nerve paralysis. Since paradoxical movement of the paralysed hemidiaphragm, if present, may be diminished or abolished by the added pneumoperitoneum (Mitchell, Hiatt, McCain, Easom, and Thomas, 1947), the combined procedure can also be considered dynamically superior to a phrenic nerve paralysis alone.

The compensatory rib movement which commonly occurs on the side of a phrenic nerve paralysis (Hoover, 1913 ; Head, 1930) is a complicating factor which, while not affecting the principle, will modify the degree of the static and dynamic changes.

Whereas there was only one case $\left(2 \frac{1}{2} \%\right)$ with no shift to the left after right, there were nine cases $\left(22 \frac{1}{2} \%\right)$ with no shift to the right after left phrenic paralysis and pneumoperitoneum.

A statistical analysis of the mean and variances of the mediastinal shift whether expressed in centimetres or as a percentage reduction of the width of the hemithorax gave the following results. 
The difference between the average mediastinal shift after left phrenic nerve crush and that after right phrenic nerve crush is not significant. The ratio between the two variances ( 4.26 for the shift in centimetres and 4.18 for the percentage reduction) is significant, and it may be concluded that the much greater variation about the average, observed after left phrenic nerve crush and pneumoperitoneum, is not due merely to sampling fluctuation.

This greater range of mediastinal shift after left phrenic nerve crush and pneumoperitoneum needs explanation. The mediastinal movement after a right phrenic nerve crush and pneumoperitoneum is principally a mediastinal shift to the left with comparatively little cardiac rotation. The mediastinal movement after the left-sided therapy comprises not only mediastinal shift to the right, but also considerable cardiac rotation (Figs. 3 and 4) which in some cases is so marked that on radioscopy the ventricular pulsation can be seen behind, and even to the right of the sternum. This greater cardiac rotation is due to the encroachment of the heart on the upper surface of the left hemidiaphragm, so that elevation of the left hemidiaphragm is accompanied by cardiac rotation. It is this more marked element of cardiac rotation that results in the greater range of mediastinal movement after left phrenic nerve crush and pneumoperitoneum, which by the method of measurement adopted in this study is represented as mediastinal shift only.

\section{Effect of Pleural Adhesions}

The figures so far given have dealt with the whole 80 cases. It has been possible, however, to separate from the main series three clinically significant groups which will be considered further. These three groups are:

Group 1.-Thirteen cases known to have complete pleural obliteration, in which an artificial pneumothorax had been attempted but in which no free pleural space was found.

Group II.-Fourteen cases known to have at least extensive pleural adhesions or a partial pleural symphysis in which an artificial pneumothorax had been induced, but was abandoned because of the extent of the adhesions or the presence of a contraselective collapse. On the abandonment of the pneumothorax, more extensive adhesions usually form, and not uncommonly complete pleural obliteration results. This group, therefore, in all probability contains some cases with complete obliteration of the pleura.

Group III.-Thirteen cases in which an artificial pneumothorax was never attempted but in which there was radiological evidence of pleural thickening of a degree at least sufficient to obliterate the costo-phrenic angle. Although such cases usually have a partial or complete obliteration, there may occasionally be a completely free pleural space.

The diaphragmatic rise and mediastinal shift in each of these three groups approximated to the mean figures for the whole series (Table I). Hence, the presence of a complete or partial pleural symphysis, or of radiological evidence of pleural thickening is compatible with the occurrence both of considerable diaphragmatic rise and of mediastinal shift after phrenic nerve crush and pneumoperitoneum, a point of some clinical significance. It is of interest that Aronovitch, Caswell, and 


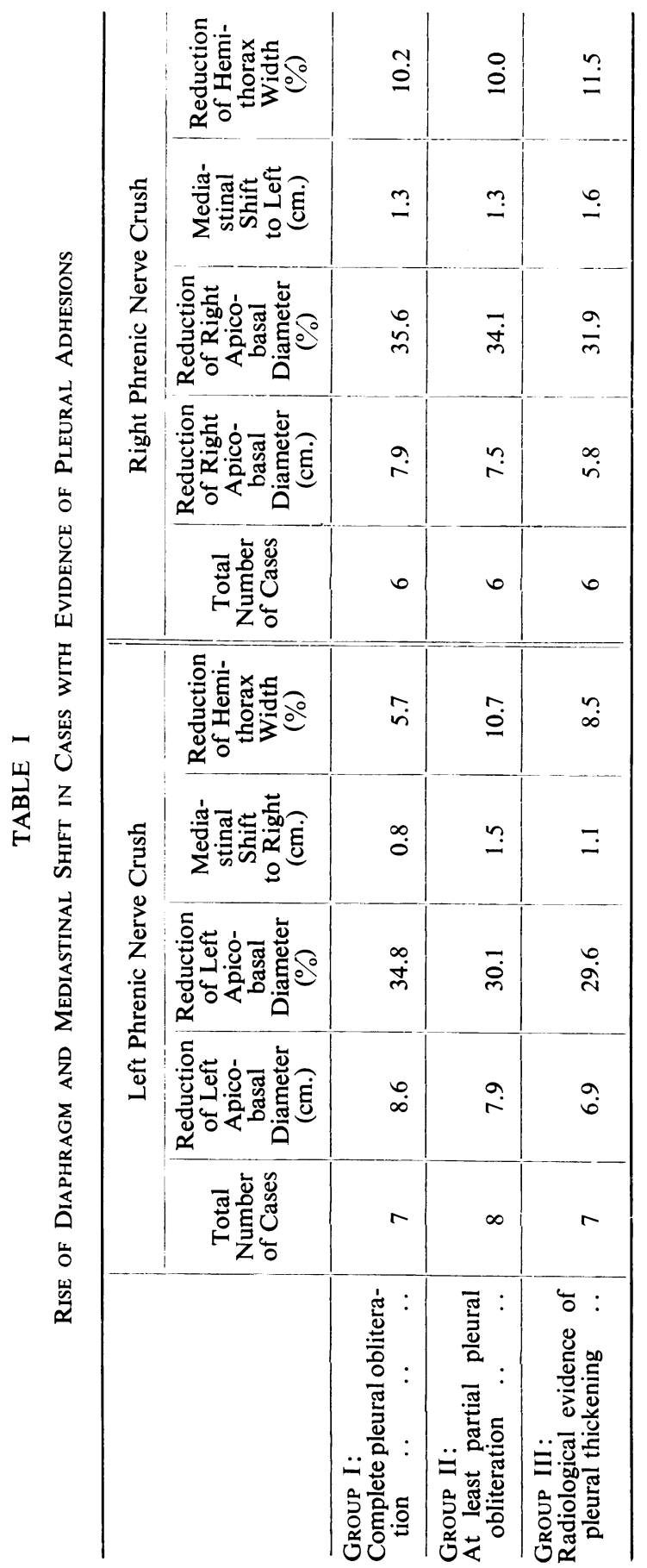


Zade (1947) and Hanrahan (1949) have reported beneficial results from this form of treatment after failure to induce a pneumothorax or after its abandonment as unsatisfactory.

\section{Tracheal ShIFT}

The mediastinal shift is not always confined to the lower mediastinum. The trachea may also be involved. In eight cases the trachea was already over towards the side of the proposed phrenic nerve crush and in four it was already towards the opposite side, leaving 68 in which it was central before treatment was begun. Among these 68 cases, it was found that the trachea moved to the right after left phrenic crush and pneumoperitoneum in 12 , and to the left after right phrenic crush and pneumoperitoneum in three. In 11 of these cases the tracheal shift was of a moderate degree and involved only the lower part, the upper part remaining central. In four, all after left phrenic nerve crush, the tracheal shift to the right was most striking (Fig. 5).

Two factors are involved in the tracheal shift. The elevation of the paralysed hemidiaphragm increases the intrapleural pressure towards atmospheric, and this will produce tracheal shift as part of the shift of the mediastinum as a whole, provided the upper mediastinum is sufficiently flexible. The radiological appearances suggest that a second factor is involved when the left hemidia phragm is paralysed. This is the rotation of the heart. One of the axes of rotation of the heart is the line between two relatively fixed points. These are the inferior vena cava below, and the termination of the aortic arch where it lies at the side of the body of the fourth thoracic vertebra. Cardiac rotation about this axis occurs when the phrenic paralysis is on the left side, and as a consequence the arch of the aorta will be moved over to the right. This rotation of the arch of the aorta, which lies to the left of the trachea, will, unless the upper mediastinum is rigid, move the lower end of the trachea to the right. Thus, the cases with striking tracheal shift also had a marked degree of cardiac rotation. After right phrenic nerve crush and pneu-

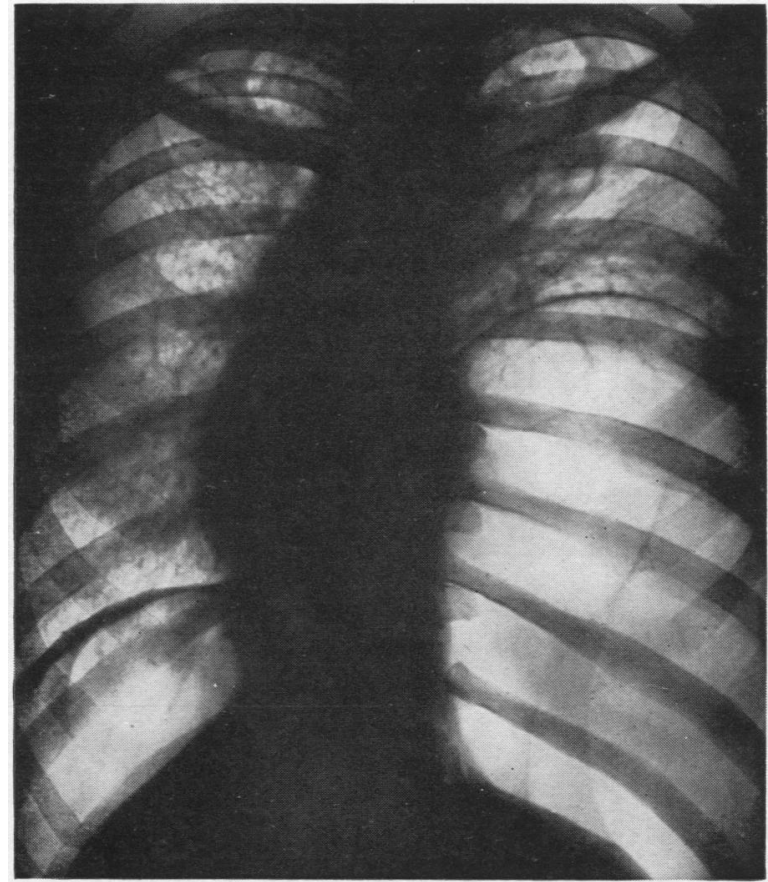

FIG. 5.-Radiograph demonstrating marked tracheal shift associated with cardiac rotation in a left phrenic nerve crush and pneumoperitoneum. The relation of the intra-abdominal air to the lower mediastinum is also evident. 
moperitoneum there is comparatively little cardiac rotation so that the aortic arch remains in its normal anatomical position, and will offer an obstruction to the movement of the trachea to the left.

Both tracheal and cardiac shift persist in the horizontal posture.

\section{The Mechanism of the Mediastinal Shift}

The lung is normally in a state of elastic tension. This is due to the fact that the minimal volume of the hemithorax (that is at maximal expiration) is larger than that of the completely relaxed lung. The elastic tension of the lung manifests itself, on the induction of an artificial pneumothorax, as a sub-atmospheric intrapleural pressure. In the presence of complete pleural obliteration, there is no measurable intrapleural pressure. Nevertheless it is patent that despite this the lung is in a state of elastic tension. In discussing the mechanism of the mediastinal shift, it is convenient to assume that there is a free pleural space and therefore to refer to the effect of the alterations of the intrapleural pressure on the position of the mediastinum. This is more convenient than referring to the elastic tension of the lung since the measurable index of elastic tension of the lung is the intrapleural pressure, and many studies of this pressure have been made under physiological, pathological, and therapeutic conditions.

In most animal species the mediastinum is very mobile so that alterations of the intrapleural pressure on the one side are closely mirrored by changes in the intrapleural pressure on the opposite side (Wright, 1945). In man, however, the mediastinum is much less mobile, and may, in the presence of, or as a result of, disease become very rigid so that the two hemithoraces become almost completely independent the one of the other as far as equalization of pressures is concerned. The two factors which determine mediastinal shift in man are (1) pressure differences in the two sides of the chest, and (2) the flexibility of the mediastinum (Pinner, 1937). It is modifications of these two factors that produce mediastinal shift in phrenic nerve crush and pneumoperitoneum therapy.

If the mediastinum is mobile, the phrenic nerve crush and pneumoperitoneum, by elevating the diaphragm and increasing the intrapleural pressure, will result in mediastinal shift towards the side of the unparalysed hemidiaphragm. Thus one factor in the mediastinal shift after phrenic nerve crush and pneumoperitoneum therapy is the rise in the intrapleural pressure on the paralysed side. The diaphragmatic rise on the unparalysed side resulting from the pneumoperitoneum will tend to increase the intrapleural pressure of its hemithorax, but because the diaphragmatic rise is smaller on this side, to a lesser degree than on the paralysed side. The net result is therefore a mediastinal movement towards the unparalysed side.

A study of these cases, however, shows that as the paralysed hemidiaphragm rises, it beds itself against the mediastinum, particularly on the right side, where there is no bulging cardiac apex (Fig. 2). The intra-abdominal air thus lies lateral to the lower part of the mediastinum being separated from it only by the thin and paralysed diaphragm. The greater the diaphragmatic rise, the larger is the area of the lateral surface of the mediastinum which comes under the influence of the pressure of the intra-abdominal air. Since in established pneumoperitoneums the intra-abdominal pressure averages $8-10 \mathrm{~cm}$. water above atmospheric, and in some 
cases attains levels as high as $15-20 \mathrm{~cm}$. water it is apparent that this high intraabdominal pressure is another factor concerned with the movement of the mediastinum away from the paralysed side, for there is no comparable area of mediastinum under the influence of the intra-abdominal pressure on the unparalysed side. Although the bulging cardiac apex will prevent such close bedding of the left hemidiaphragm, this is offset in some cases by the cardiac rotation which is often present with a left phrenic crush and pneumoperitoneum. This rotation of the cardiac apex enables the left hemidiaphragm to appose itself more closely against the side of the mediastinum (Figs. 4 and 5). Only the lower mediastinum is under the influence of the high intra-abdominal pressure. The upper mediastinum is not under its direct influence, and this contributes to the lesser tendency of the trachea and upper mediastinum, as compared with the lower mediastinum, to shift to the opposite side.

A third factor is involved. This concerns the fixity of the mediastinum, and loss of anchorage of the lower end of the mediastinum resulting from the hemidiaphragmatic paralysis. Not only does the hemidiaphragm rise, but the lower mediastinum itself moves bodily upwards on the side of the paralysed diaphragm (Figs. 2 and 5). The diaphragm has, therefore, as one of its functions the fixation of the mediastinum from below, and any reduction of this fixation will predispose to mediastinal shift. Anatomically, all the diaphragmatic muscular fibres inserted into the central tendon, whether sternal, costal, or vertebral in origin, pass upwards and inwards to the central tendon, and so, when in a state of tonus, are capable of anchoring the half of the central tendon into which they are inserted. The most efficient part of the diaphragm in producing anchoring of the central tendon is, however, the crural portion. The right crus arises from the bodies of the first three lumbar vertebrae and their intervertebral discs, and the left crus from the corresponding parts of the upper two only. These fibres pass upwards and forwards to the central tendon and exert an almost vertical pull on it. Since the pericardium is attached to the upper surface of the central tendon, paralysis of either crus will diminish the fixity of the mediastinum from below. Once a hemidiaphragm is paralysed, the mediastinum loses one of its anchors and so moves bodily upwards on that side. It swings round the attachment of the other crus, a movement which is increased by inequalities of pressure on its two sides.

A further sub-diaphragmatic anchorage is provided by the inferior vena cava on the right side. That this too is capable of considerable elongation is shown by the radiological appearances of cases with a right hemidiaphragmatic paralysis and pneumoperitoneum. Here, there is often a considerable interval between the superior surface of the liver and the diaphragm, across which the inferior vena cava passes.

\section{The Therapeutic Significance of the Mediastinal Shift}

Although it is not within the scope of this study to discuss the therapeutic results of phrenic nerve crush and pneumoperitoneum therapy of pulmonary tuberculosis the therapeutic significance of the mediastinal shift merits consideration.

If the lesion in the worse affected lung is adequately controlled by an artificial pneumothorax, lesions in the "good" lung often improve considerably or may 
even heal completely. Similarly in phrenic nerve crush and pneumoperitoneum therapy, although the greater effect can be expected to occur on the side of the phrenic nerve crush, improvement, and even healing, often occurs on the unparalysed side. Several factors appear to play a part in the improvement of the "better" lung. These are: (1) adequate control of the active disease in the worse lung improves the general condition and enables the patient to combat the disease in the "good" lung more effectively. (2) The patient with a phrenic nerve crush and pneumoperitoneum may have a period of several weeks lying flat on his back with the foot of the bed raised, to be followed by several weeks or even months of semi-absolute bed rest during the establishment of the pneumoperitoneum. This rest factor may also have a beneficial effect on all the pulmonary lesions. (3) The reduction of the apico-basal diameter on the unparalysed side may be quite considerable and so can be considered as a factor producing a degree of pulmonary collapse on the unparalysed side. (4) The mediastinal shift towards the "good" lung by reducing the lung volume and diminishing the elastic tension of the lung would also act as a collapse measure for the contralateral lung.

All these factors may be expected to contribute towards the retrogression of disease in the better lung. That mediastinal shift can be a significant factor is demonstrated by reference to other forms of collapse therapy, of which contralateral artificial pneumothorax will be considered first, for although this treatment never found general favour, it still illustrates the significance of mediastinal shift.

Contralateral artificial pneumothoraces were first used by Ascoli (1929). They were induced in patients with unilateral disease in whom pleural obliteration prevented the induction of an artificial pneumothorax on the diseased side. In the presence of a mobile mediastinum it was found that the induction of an artificial pneumothorax on the side of the healthy lung was capable of producing improvement in the diseased lung, and several writers have since confirmed this (Jacono, 1929 ; Fichera and Giuffrida, 1930 ; Lucacer, 1931). In these cases the first and third factors can be excluded and the factors of rest and mediastinal shift remain to explain the improvement of the disease. This is, therefore, evidence in favour of the importance of mediastinal shift in producing improvement in intra-pulmonary disease.

Further evidence can be adduced from patients being prepared for a thoracoplasty in which it has been found necessary, as a preliminary, to induce an artificial pneumothorax to control disease in the "better" lung. In these cases, the establishment of the pneumothorax is sometimes followed by further retrogression of the lesion in the worse lung and even reduction in the size of cavities, if there is a mobile mediastinum. Such cases have as a rule already had months of bed rest and have ceased to improve radiologically. Any further improvement following the artificial pneumothorax can therefore reasonably be related to the attendant mediastinal shift rather than to further bed rest.

It is hardly necessary to add that lesions in the contralateral lung may be unaffected by phrenic nerve crush and pneumoperitoneum therapy, and indeed deterioration and even contralateral spread may occur during the course of this therapy. 


\section{SUMMARY}

The reduction of the apico-basal diameters of the lungs and the mediastinal shift following phrenic nerve crush and pneumoperitoneum therapy of pulmonary tuberculosis was studied radiologically in 80 patients, 40 with a left phrenic nerve crush and pneumoperitoneum, and 40 with a right phrenic nerve crush and pneumoperitoneum.

The mediastinal movement may include tracheal shift and there is often considerable cardiac rotation particularly after left phrenic paralysis. The mediastinal shift is present even with the patient lying down.

The mediastinal shift is due to three factors. These are, first, the loss of fixation of the lower end of the mediastinum resulting from the hemidiaphragmatic paralysis; second, the increase in the intrapleural pressure due to the considerable diaphragmatic rise on the paralysed side ; and third, the pressure of the intraabdominal air which lies lateral to the lower end of the mediastinum on the side of the paralysed hemidiaphragm.

The reduction of the apico-basal diameter on the unparalysed side and the mediastinal shift towards this side are both collapse measures for the "good" lung.

The combined phrenic nerve crush and pneumoperitoneum therapy has a mechanically superior effect on the diseased lung to a phrenic nerve paralysis alone. The effect of the pneumoperitoneum alone on the unparalysed side is mechanically inferior to a phrenic nerve paralysis.

Diaphragmatic elevation occurs whether the pleura is free, partially or completely obliterated, or thickened.

I am indebted to Dr. F. Temple Clive, Physician-Superintendent of Preston Hall Hospital, and Dr. L. R. J. Rinkel, Medical Superintendent of Nayland Sanatorium, for permission to use the clinical material, to Dr. T. V. Crichlow for his advice on the radiological aspects, to Mr. P. Armitage, of the Statistical Research Unit of the Medical Research Council, for his advice on the statistical significance of the figures, and to Professor F. R. G. Heaf for his criticisms of this paper.

\section{REFERENCES}

Agnello, V. (1938). Ann. Ist. C. Forlanini, 2, 437.

Aronovitch, M., Caswell, L. A., and Zadé, J. A. (1947). Canad. med. Ass. J., 57, 122.

Ascoli, M. (1929). Dtsch. med. Wschr., 55, 1369.

Benatt, A. J., and Berg, W. F. (1945). Amer. Heart J., 30, 579.

Elwood, B. L., Piltz, G. F., and Potter, B. P. (1939). Bull. Amer. Acad. Tuberc. Physicians, 3, 140.

Fichera, S., and Giuffrida, F. (1930). Rev. Tuberc., 11, 528.

Hanrahan, M. (1949). Irish J. med. Sci., 6S, 112.

Hansen, O. S., and Maly, H. W. (1934). Amer. Rev. Tuberc., 30, 527.

Head, J. R. (1930). Surg. Gynec. Obstet., 50, 929.

Hoover, C. F. (1913). Arch. intern. Med., 12, 214.

Jacono, V. (1929). Riv. Pat. Clin. Tuberc., 3, 989.

Lucacer, M. (1931). Amer. Rev. Tuberc., 24, 50.

Mitchell, R. S., Hiatt, J. S., McCain, P. P., Easom, H. F., and Thomas, C. D. (1947). Amer. Rev. Tuberc., 55, 307.

Nehil, L. W., and Alexander, J. (1933). J. thorac. Surg., 2, 549.

Pinner, M. (1937). In Alexander's Collapse Therapy of Pulmonary Tuberculosis. Charles C. Thomas \& Co. Springfield, Illinois. P. 65.

Trimble, H. G., Eaton, J. L., Crenshaw, G. L., and Gourley, I. (1948). Amer. Rev. Tuberc., 57, 433.

Wright, S. (1945). Applied Physiology. 8th Edition. P. 590 . Oxford University Press. London. 